Supporting Information

\title{
SWITCHABLE FRICTION OF STIMULUS-RESPONSIVE HYDROGELS
}

\author{
Debby P. Chang, John E. Dolbow, Stefan Zauscher
}

Derivation of the friction/torque equation:

The frictional force, $F$, is determined from the measured torque, $T$,

$$
F=4 T / 3 R
$$

where $R$ is the radius of contact area. ${ }^{1}$ It is derived under two assumptions: first, that the gel friction shear force is linearly proportional to the contact area under a constant normal pressure; second, the friction per unit area is linearly proportional to shear velocity.

The rotational velocity varies with distance from the center of the axis; therefore, friction per unit area at a distance $r$ from the axis is,

$$
f=c \omega r
$$

where $c$ is a constant independent of $r$. The total frictional force is then simply obtained by integration of the frictional force per unit area over the whole circle,

$$
F=\int_{0}^{R} 2 \pi r \cdot f \cdot d r=\frac{2}{3} \pi c \omega R^{3},
$$

and the total torque from friction is given by,

$$
T=\int_{0}^{R} 2 \pi r \cdot f \cdot r \cdot d r=\frac{1}{2} \pi c \omega R^{4}
$$

Friction can then be calculated from the measured torque with Equation 1. 

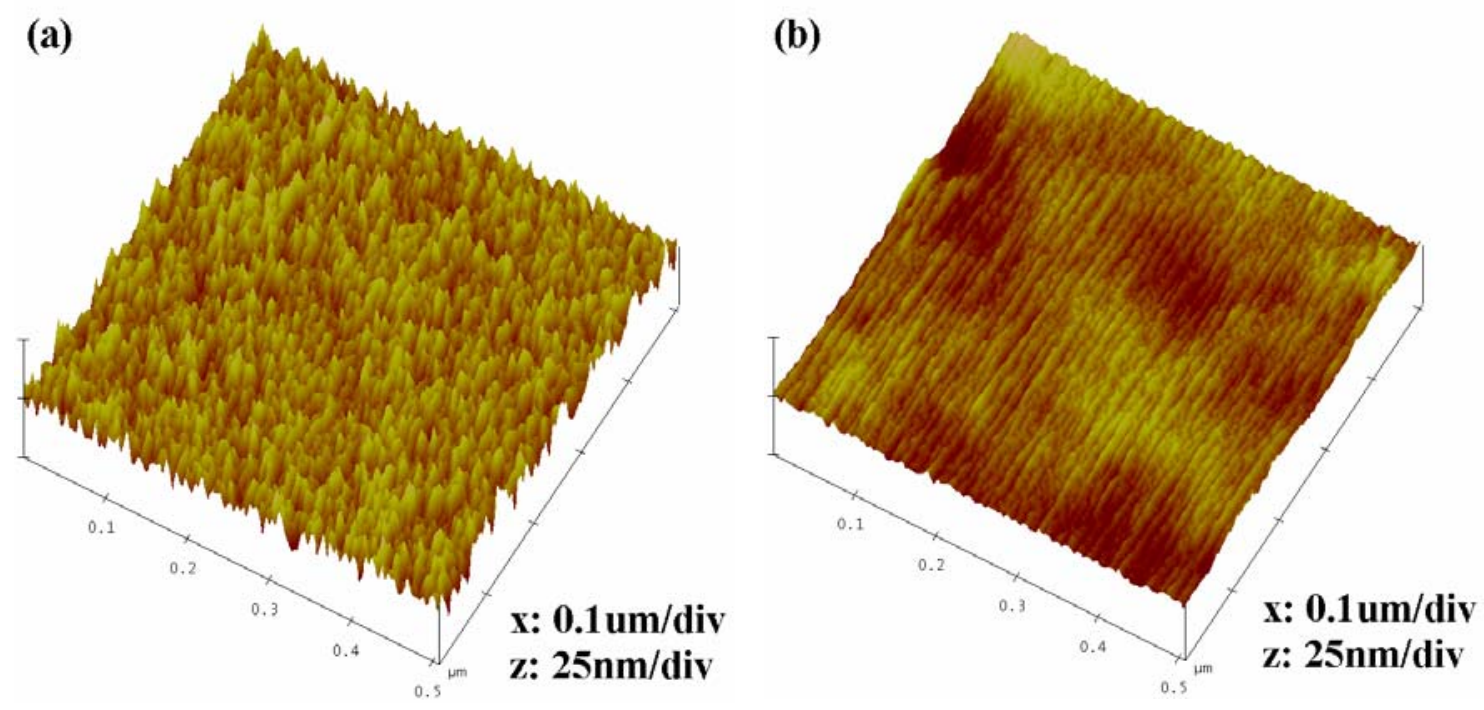

Figure 1. Three-dimensional tapping mode AFM images $(0.5 \mu \mathrm{m} \times 0.5 \mu \mathrm{m})$ of pNIPAAm gel in (a) the swollen and (b) the collapsed state. RMS roughness is $2.9 \mathrm{~nm}$ for the swollen gel and $1.3 \mathrm{~nm}$ for the collapsed gel.

Although the roughness of the gel in the swollen state, as probed by the AFM cantilever tip, is larger than for the gel in the collapsed state, the image is essentially flat (Figure 1a). In the collapsed state, the roughness is smaller, but an underlying corrugation of the surface is clearly visible. We believe that it is this corrugation which becomes more pronounced when one considers larger image scan sizes (see Figure $4 \mathrm{~b}$ in the paper) that is important for friction in the sliding gels. The image of the surface of the swollen gel contains a significant contribution from probe-surface adhesion interactions, giving rise to the larger apparent roughness. For the gel in the collapsed state, dangling polymer chain ends and chain segments are collapsed, explaining the overall smaller roughness. However, the surface features in the collapsed gel have "coarsened" when compared with those in the swollen state. This observation is in agreement with previous findings on the surface topography of pNIPAAm gels. ${ }^{2,3}$ 


\section{References}

(1) Gong, J. P.; Iwasaki, Y.; Osada, Y.; Kurihara, K.; Hamai, Y., J. Phys. Chem. B 1999, 103, (29), 6001-6006.

(2) Suzuki, A.; Yamazaki, M.; Kobiki, Y., J. Chem. Phys. 1996, 104, (4), 1751-1757.

(3) Suzuki, A.; Yamazaki, M.; Kobiki, Y.; Suzuki, H., Macromolecules 1997, 30, (8), 2350-2354. 